\title{
Degeneracy in One Dimensional Quantum Systems: Dynamically Shifted Oscillator
}

\author{
Pirooz Mohazzabi ${ }^{1 *}$, G. Clark Alexander ${ }^{2}$ \\ ${ }^{1}$ Department of Mathematics and Physics, University of Wisconsin-Parkside, Kenosha, WI 53141, USA. \\ 2 Department of Mathematical Sciences, DePaul University, Chicago, IL 60604, USA. \\ * Corresponding author. Tel.: (262) 595-2529; email: mohazzab@uwp.edu \\ Manuscript submitted June 2, 2016; accepted November 2, 2016. \\ doi: 10.17706/ijapm.2017.7.2.79-86
}

\begin{abstract}
We study the dynamically shifted quantum oscillator in one dimension, whose detailed examination reveals some very interesting and unexpected results. While several articles have been written on the classical dynamically shifted oscillator, the quantum version is somewhat more difficult since it defies solution by standard algebraic and analytic techniques. In this work, we show that the analytic approach to this problem results in a trivial, non-normalizable, and thus nonphysical solution. Therefore, we undertake a numerical approach and find two strikingly different results. First, the energy eigenvalues rise when the dynamic shift is positive, which is almost intuitive. The second result is surprising as the system shows degeneracy in one dimension despite the fact that this potential violates two of the three required criteria described previously by other investigators. This work, therefore, opens a door to finding multiple classes of one-dimensional degenerate systems with continuous, symmetric potentials.
\end{abstract}

Key words: Degeneracy, one dimension, quantum, dynamically shifted, oscillator.

\section{Introduction}

The quantum dynamically shifted oscillator in one dimension is given by the potential

$$
V(x)= \begin{cases}\frac{1}{2} k\left(x+x_{0}\right)^{2}, & x \geq 0 \\ \frac{1}{2} k\left(x-x_{0}\right)^{2}, & x<0\end{cases}
$$

which reduces to the simple harmonic oscillator for $x_{0}=0$. For $x_{0}>0$ and $x_{0}<0$ the potential is continuous but not smooth at the origin, as shown in Fig. 1. In this article we show that several unusual phenomena occur when $x_{0}$ is small negative. For $x_{0}=0$ the solution is well known and perhaps the most widely studied example in one-dimensional quantum mechanics, while the case of $x_{0}>0$ simply excites the ground state. The unusual phenomena that occur when $x_{0}$ is small negative are two-fold. First, the eigenvalues fall below the ground state value of $1 / 2 \hbar \omega$ as if the dynamic shift locally produces an anharmonic shift similar to the linear case, $\lambda x$. Second, when the reduced dynamic shift $\xi_{0}$ becomes smaller than approximately -3 , we begin seeing degeneracy in eigenvalues producing two solutions of opposite parity.

The question of degeneracy in one dimension is interesting as most of the literature explores higher dimensional degeneracy. In their paper, Kar and Parwani [1] explore the possibility of degenerate bound states in one dimensional systems. Along with Koley and Kar [2], they present the following bottomless 
potential,

$$
V(x)=-\left(A_{1} \cosh ^{2 v} x+A_{2} \operatorname{sech}^{2} x\right)
$$

with $v>0$ and $A_{2}=\frac{v}{2}\left(\frac{v}{2}+1\right)$, as giving two eigenstates of different parity,

$$
\begin{aligned}
& \psi_{1}(x)=\frac{\cos \left(\sqrt{A_{1}} \int \cosh ^{v} x d x\right)}{\cosh ^{v / 2} x} \\
& \psi_{1}(x)=\frac{\sin \left(\sqrt{A_{1}} \int \cosh ^{v} x d x\right)}{\cosh ^{v / 2} x}
\end{aligned}
$$

These potentials, however, do not appear in elementary quantum systems, but brane scenarios. The potential that we investigate, however, is very similar to that experienced by, for example, the Nitrogen atom in an Ammonia molecule [3].
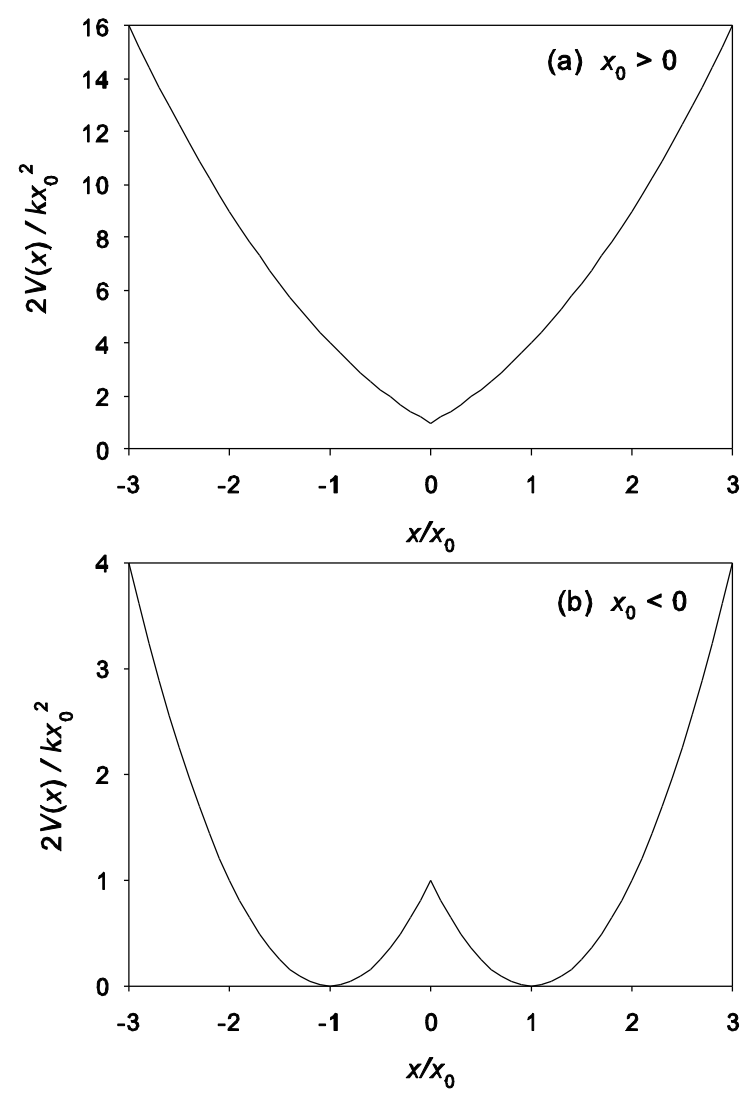

Fig. 1. Dimensionless plots of the dynamically shifted oscillator for positive and negative dynamic shifts.

\section{The Mathematics of the Dynamically Shifted Oscillator}

The standard method for solving a piecewise potential analytically is to solve each piece separately and then match the solutions. In particular this technique has been applied not only in textbooks, but in numerous articles involving oscillators with altered potentials, for example the biharmonic oscillator [4] and the harmonic plus a delta-potential [5].

By the postulates of quantum mechanics, wave function solutions to equations with continuous potentials are required to be continuous and have continuous first and second derivatives. Every derivative which 
exists in the potential energy function requires another continuous derivative in the wave function solution. Therefore, in the case of the dynamically shifted oscillator we must have at least two continuous derivatives. The problem one experiences with trying to find a global solution is that the solutions to each half are well known as the harmonic oscillator solutions. When $x_{0} \neq 0$ one finds that matching solutions yields $0=0$ which is, of course, true but not useful.

To be explicit about this solution, let us define the following unitless quantities

$$
\xi=\left(\frac{m \omega}{\hbar}\right)^{1 / 2} x, \quad \xi_{0}=\left(\frac{m \omega}{\hbar}\right)^{1 / 2} x_{0}, \quad \varepsilon=\frac{E}{\hbar \omega}
$$

The reduced time-independent Schrödinger equation has the solutions

$$
\psi_{n}(x)=( \pm 1)^{n} A_{n} \exp \left[-\frac{\left(\xi \pm \xi_{0}\right)^{2}}{2}\right] H_{n}\left(\xi \pm \xi_{0}\right), \quad n=0,1,2, \ldots
$$

where $H_{n}$ are Hermite polynomials and $A_{n}$ are constants. The upper and lower signs hold for $\xi \geq 0$ and $\xi<0$, respectively, and the corresponding eigenvalues for each branch are

$$
\varepsilon_{n}=n+\frac{1}{2}, \quad n=0,1,2, \ldots
$$

Now matching each side of the solution we see that the following equations must be satisfied

$$
\begin{aligned}
& A_{2 k} H_{2 k}\left(\xi_{0}\right) \sinh \left(\xi_{0}^{2}\right)=0 \\
& A_{2 k+1} H_{2 k+1}\left(\xi_{0}\right) \cosh \left(\xi_{0}^{2}\right)=0
\end{aligned}
$$

These equations, however, are satisfied if and only if $A_{n}=0$ for all $n$ unless $\xi_{0}=0$. This clearly implies that no global analytic eigenfunction can exist except for $\xi_{0}=0$ (which is the case of the harmonic oscillator). The way in which one may guarantee global analytic solutions is to have a smooth potential. It is, of course, possible to have a smooth solution via matching solutions, but there is no guarantee that these will give analytic solutions. The only mathematical guarantee is to have a smooth potential.

If one were to attempt to solve this algebraically, the standard approach of factoring the differential operator in raising and lowering operators works exactly as expected. Essentially, there is no detectable difference from the standard textbook treatment of the harmonic oscillator aside from having shifted the minimum of the potential. The algebraical analysis predicts that we should recover the same eigenvalues as the unshifted oscillator, which we can falsify via numerical techniques given in the succeeding sections.

The main problem in the solution of the dynamically shifted oscillator is that the analytic techniques for solution do not account for the (unitless) eigenvalues dipping below the ground state value $\varepsilon_{0}=1 / 2$. In fact, none of the algebraic or analytic techniques show any sign of eigenvalues being dependent upon the value of the dynamic shift. Numerically, however, this is what happens. The problem here is that we are dealing with a system which appears innocuous, but Hartmann [6] and Mohazzabi [7] have shown that this has an intrinsically different behavior.

\section{Degeneracy Conditions in One Dimension}

Kar and Parwani [1] explore degenerate bound states in one dimensional quantum mechanics with the additional restriction that a potential be non-singular in any finite domain. Of course, the last stipulation is what makes the question difficult. It is rather easy to simply place two infinite potential wells some distance apart on the real line and solve the Schrödinger equation in each part then flip the sign of one of the 
solutions. Moreover, many systems with an infinite singularity in a finite domain exhibit this kind of degeneracy. The paper of Kar and Parwani, however, asks a much more interesting question. The conclusion they draw is that a one-dimensional quantum system cannot be degenerate unless the following criteria are met:

1) $V(x)$ is a continuous function.

2) $V(x)$ is unbounded from below at $\infty$.

3) The eigenfunction $\psi$ has infinite derivative as $x \rightarrow \infty$.

The third criterion emphasizes that the momentum is divergent, which corresponds to divergent kinetic energy. The only way to maintain a finite total energy is to have unbounded negative potential energy. While the constructions given by Kar and Parwani [1] maintain all these criteria, we will show that there is yet another degenerate bound state system with continuous potential and smooth wave functions with convergent momentum, but with a potential bounded from below, although unbounded from above. Thus, this system meets the first criterion stipulated above, but violates the other two. If we were to replace criterion one with the requirement that $V(x)$ be smooth, then the dynamically shifted oscillator as defined currently would fail to meet this criterion as well. However, it is possible to replace the potential listed in equation (1) with a smooth potential which gives identical numerical results to arbitrary accuracy.

\section{Energy Eigenfunctions and Eigenvalues of the Dynamically Shifted Oscillator}

Given that the algebraic and analytic techniques do not explain the dip in the energy eigenvalue, the authors undertake a numerical study of eigenvalues and eigenfunctions for the dynamically shifted oscillator.

In Fig. 2 we see $\psi_{0}$ corresponding to different values of the reduced shift $\xi_{0}$. Notice how the eigenfunction splits into two peaks as $\xi_{0}$ decreases from 1 to -3 . An interesting effect we see is that the corresponding eigenvalues behave as we might expect when $\xi_{0}=1$, that is, $\varepsilon_{0}=1.4999$. As we anticipate when the potential energy is raised by one unit (over that of the harmonic oscillator), the energy eigenvalue rises by one. However, when $\xi_{0}$ decreases to -1 and -2 the energy eigenvalues drop below the standard ground state solution of $\varepsilon_{0}=1 / 2$. As we decrease $\xi_{0}$ further to -3 , we nearly recover the original ground state eigenvalue of the harmonic oscillator.

Turning to Fig. 3, we see the evolution of the first excited state $\psi_{1}$ as a function of the reduced dynamic shift. Similar to the ground state, $\psi_{1}$ appears to split into two separate waves as $\xi_{0}$ becomes increasingly negative. The figure, however, does not seem to show the corresponding eigenvalues hitting a minimum. Notice the peculiarity happening with $\varepsilon_{1}$ as $\xi_{0}$ becomes increasingly more negative. Looking to Figure 6 , we see that $\varepsilon_{0}$ and $\varepsilon_{1}$ both approach $1 / 2$ but $\varepsilon_{0}$ from below and $\varepsilon_{1}$ from above.

Evolutions of $\psi_{2}$ and $\psi_{3}$ as a function of the reduced dynamic shift $\xi_{0}$ show similar behavior. As the reduced dynamic shift decreases form 1 to -3 , each eigenfunction splits into two branches. The values of $\varepsilon_{2}$ and $\varepsilon_{3}$ exhibit markedly similar behaviors to those of $\varepsilon_{0}$ and $\varepsilon_{1}$, respectively, but with both values now approaching 1.5 .

Turning our attention to Fig. 4, we begin to see degenerate behavior. When our dynamic shift is "large" negative, in this case $\xi_{0}=-5$, then we see $\varepsilon_{0}=0.5$ and there are two eigenfunctions, one of even parity and one of odd parity. However, both eigenfunctions are locally even about $\pm \xi_{0}$.

Fig. 5 shows the same situation for $\psi_{1}$ and again there are two eigenfunctions, one of even parity and one of odd parity, both corresponding to the same eigenvalue $\varepsilon_{1}=1.5$. In this case, however, both eigenfunctions are locally odd about $\pm \xi_{0}$.

Further investigation of the higher eigenfunctions and eigenvalues promulgate the evidence that the 
dynamically shifted oscillator does indeed exhibit degenerate behavior. Following suit from Figures 4 and 5 we see the that eigenfunctions $\psi_{2 k}$ are locally even and $\psi_{2 k+1}$ are locally odd about $\pm \xi_{0}$.
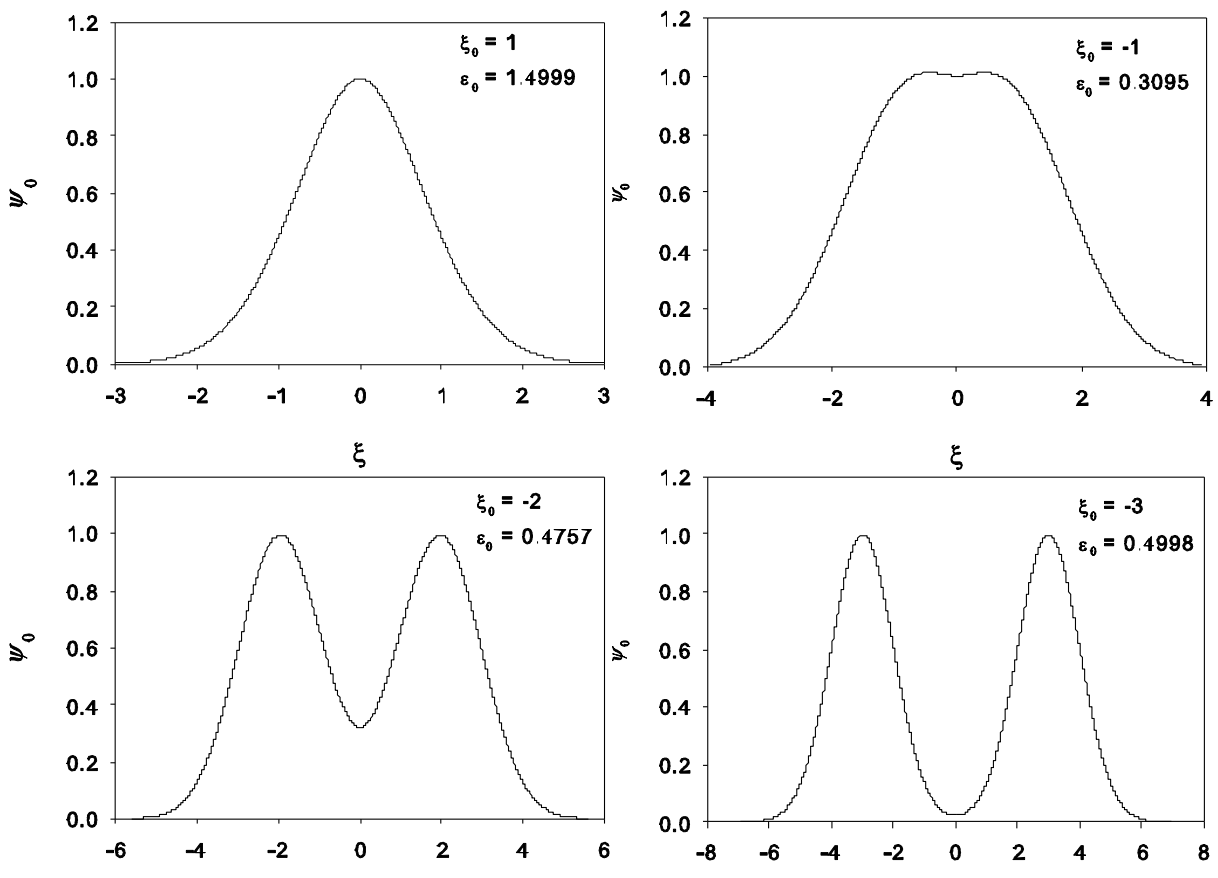

$\xi$

$\xi$

Fig. 2. Ground-state eigenfunction of the dynamically shifted oscillator for different values of the dynamic shift $\xi_{0}$.
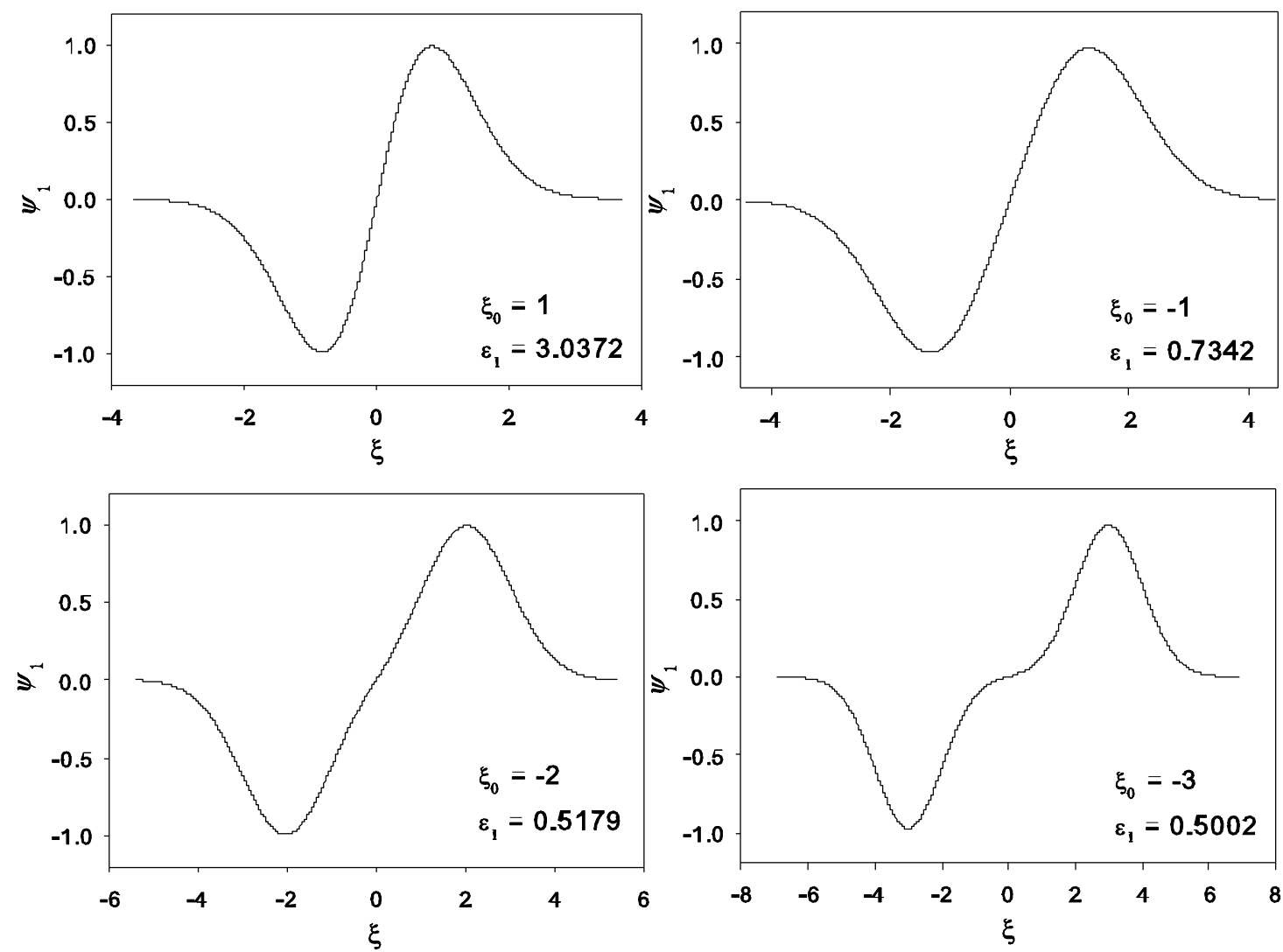

Fig. 3. The first excited state eigenfunction of the dynamically shifted oscillator for different values of the dynamic shift $\xi 0$. 
Finally, Fig. 6 explicitly reveals how the eigenvalues split. The eigenvalues of the dynamically shifted oscillator bifurcate with respect to the dynamic shift. The "upper" degenerate eigenvalues correspond to the odd eigenfunctions, whereas the "lower" eigenvalues correspond to the even eigenfunctions.
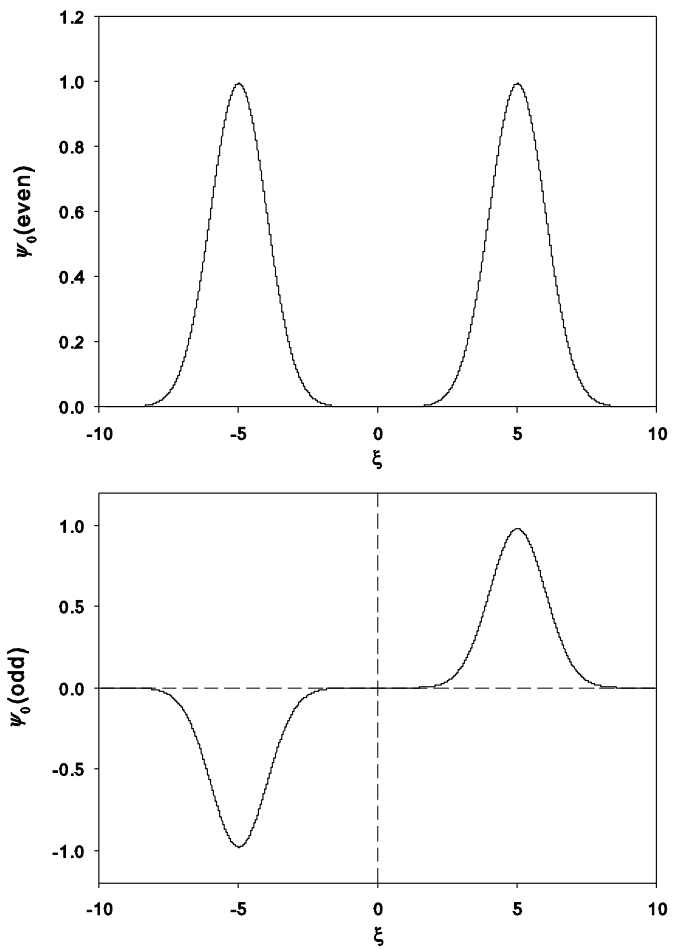

Fig. 4. The even and odd ground state eigenfunctions of the dynamically shifted oscillator for a "large" negative dynamic shift, in this case $\xi_{0}=-5$. Note that both eigenfunctions are "locally" even.
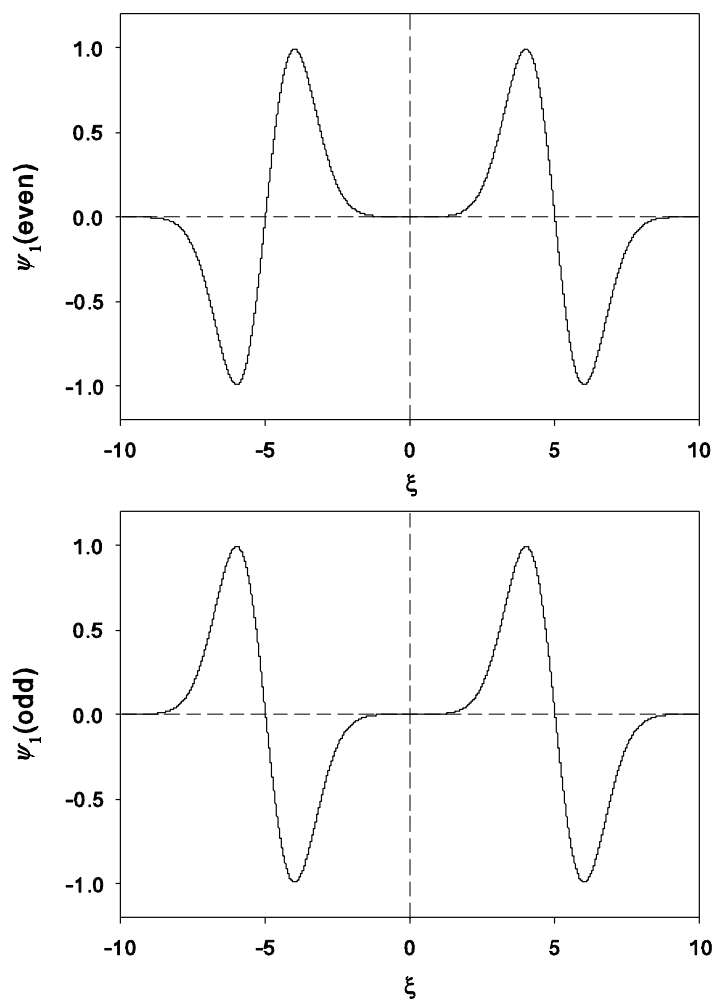

Fig. 5. The even and odd first excited state eigenfunctions of the dynamically shifted oscillator for a "large" negative dynamic shift, in this case $\xi_{0}=-5$. Note that both eigenfunctions are "locally" odd. 


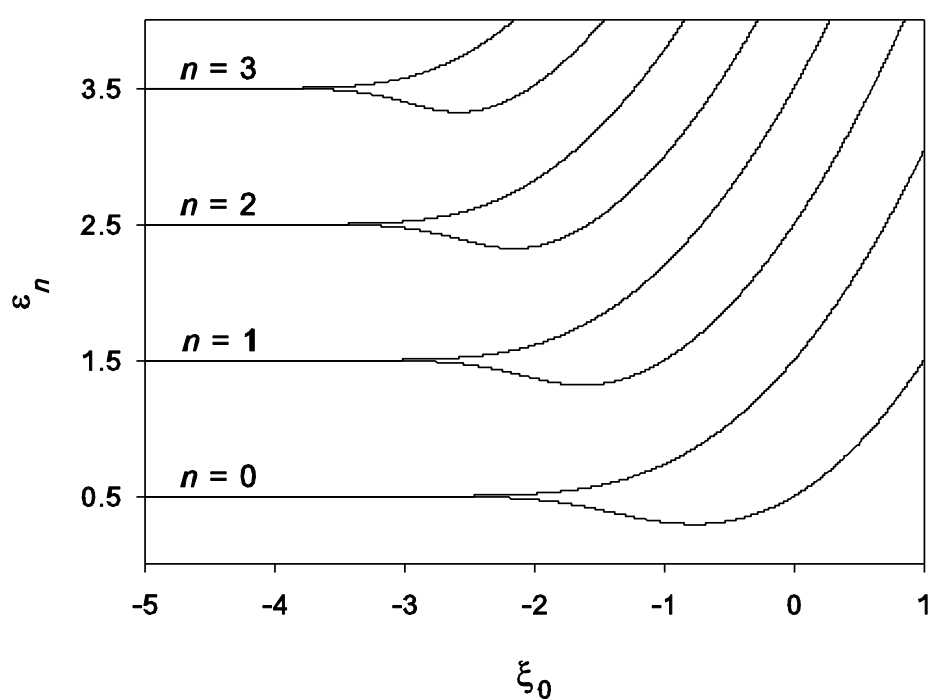

Fig. 6. Bifurcation of energy eigenvalues of the dynamically shifted oscillator with respect to the dynamic shift.

\section{Conclusion}

The dynamically shifted oscillator is an interesting quantum mechanical system in one dimension for several reasons. Of primary importance is that it exhibits a degeneracy in eigenvalues in one dimension when $\xi_{0} \approx-3$. What's interesting about this is the fact that the standard algebraic techniques do not predict any difference in energy from the standard harmonic oscillator. Moreover, the dynamic shift when negative takes nondegenerate states and adiabatically moves them into degenerate states. One remaining question to be resolved, since we have seen the numerical solution, is whether we have a true degeneracy or a pseudo-degeneracy. In this case, the question becomes rather meaningless since the algebraic solution suggests the all states are degenerate. Numerically, we can render this question irrelevant since we may in fact have a separation of eigenvalues, but when $\xi_{0}$ becomes large enough (negatively) then we will have eigenvalues which are arbitrarily close in value. Furthermore, in a physical sense, the energy eigenvalues certainly fall within the uncertainty limit and are therefore physically indistinguishable. In addition to this, the potential can be made smooth by replacing the given potential on $[-\delta, \delta]$ with an arbitrarily high degree (but finite) Fourier expansion which matches our value at $V( \pm \delta)$ so as to guarantee smooth solutions with degenerate eigenvalues. Finally, this system opens the door to finding multiple classes of one-dimensional degenerate systems with continuous, symmetric potentials.

\section{References}

[1] Kar, S., \& Parwani, R. R. (2007). Can degenerate bound states occur in one-dimensional quantum mechanics? Europhysics Letters, 80(3), 30004.

[2] Koley, R., \& Kar, S. (2007). Exact bound states in volcano potentials. Physics Letters A, 363, 369-373.

[3] Eisberg, R., \& Resnick, R. (1985). Quantum Physics of Atoms, Molecules, Solids, Nuclei, and Particle (2nd ed.). New York: Wiley.

[4] Gettys, W. E., \& Graben, H. W. (1975). Quantum solution for the biharmonic oscillator. American Journal of Physics, 43, 626-629.

[5] Janke, W., \& Cheng, B. K. (1988). Statistical properties of a harmonic plus a delta-potential. Physics Letters $A, 129,140-144$.

[6] Hartmann, W. M. (1986). The dynamically shifted oscillator. American Journal of Physics, 54, 28-32. 
[7] Mohazzabi, P. (2004). Theory and examples of intrinsically nonlinear oscillators. American Journal of Physics, 72, 492-498.

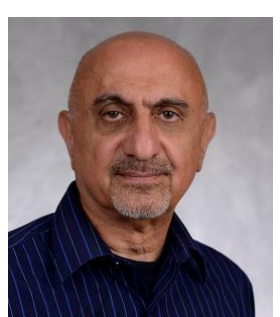

simulations.

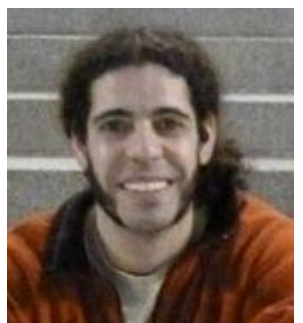

Pirooz Mohazzabi received his $\mathrm{PhD}$ in materials science and engineering from University of California, Berkeley, in1975. He joined University of Wisconsin-Parkside in 1986, where he is currently a professor of physics. He has published extensively in a wide variety of areas, ranging from bicycle stability to cancer theory. His current research focuses on thermodynamics and statistical mechanics of small systems, using theoretical and computational methods including molecular dynamics and Monte Carlo

Clark Alexander received his $\mathrm{PhD}$ in mathematics from Northwestern University in 2008. He is currently a visiting assistant professor at DePaul University in Chicago, where he has been on faculty since 2011. His research interests are mathematical physics, noncommutative geometry, and quantum algorithms. 